\title{
System Identification of a Nonlinear Mode for the Shuttle Radar Topography Mission
}

\author{
Paul B. Brugarolas, ${ }^{*}$ David S. Bayard, ${ }^{\dagger}$ John T. Spanos, ${ }^{\ddagger}$ and William G. Breckenridge ${ }^{\S}$ \\ Jet Propulsion Laboratory, California Institute of Technology, Pasadena, California 90012
}

\begin{abstract}
A study is presented to identify a nonlinear bending mode for a $60-\mathrm{m}$ space structure. This study was done in support of the Shuttle Radar Topography Mission (SRTM) and postflight height reconstruction efforts. For this purpose, one linear model and three nonlinear models of the structural mode were considered and evaluated. The best model was determined based on in-flight data collected during the mission and was implemented as part of the final ground software that was used for reconstructing relative radar antenna motion for the SRTM interferometer payload. High accuracy estimates of the relative states were essential for supporting the motion compensation algorithm used in the radar interferometry processor for calculating the desired topographic maps. The improvement resulting from identifying nonlinear modal behavior contributed to meeting mission performance requirements.
\end{abstract}

\section{Introduction}

$\mathbf{I}$ N February 2000, the Shuttle Radar Topography Mission (SRTM) was flown onboard the Space Shuttle Endeavor to collect topographic data of the Earth (between $60^{\circ}$ north latitude and $54^{\circ}$ south latitude). The SRTM mission was jointly sponsored by NASA and the National Geospatial-Intelligence Agency with a goal of creating the first near-global high-resolution topographic maps of the Earth.

To acquire the topographic data, SRTM used a technique called radar interferometry. This technique is capable of extracting elevation data from differences between two radar images taken from two different locations, assuming that the relative position and orientations of the radar antennas are known with respect to the surface. For this purpose the SRTM payload had two sets of radar antennas (one on the shuttle bay and one at the end of a $60-\mathrm{m}$ deployable mast) and a suite of high-precision instruments, denoted as the attitude and orbit determination avionics (AODA), to measure the position and orientation of the radar antennas with respect to the Earth. Globalpositioning-system receivers measured the position of the payload with respect to the Earth surface, and a star tracker and an inertial reference unit were used to measure the orientation of the antenna located on the shuttle bay. To measure the relative orientation and position of the outboard antenna located at the tip of the $60-\mathrm{m}$ boom with respect to the shuttle, a target tracker and a laser rangefinder were used.

The target tracker measured the relative orientation by tracking an array of light-emitting diodes (LEDs) mounted on the outboard an-

Presented as Paper 2002-4937 at the AIAA Guidance, Navigation, and Control Conference, Monterey, CA, 5-8 August 2002; received 10 December 2003; revision received 6 October 2004; accepted for publication 6 November 2004. Copyright (C) 2005 by the American Institute of Aeronautics and Astronautics, Inc. The U.S. Government has a royalty-free license to exercise all rights under the copyright claimed herein for Governmental purposes. All other rights are reserved by the copyright owner. Copies of this paper may be made for personal or internal use, on condition that the copier pay the $\$ 10.00$ per-copy fee to the Copyright Clearance Center, Inc., 222 Rosewood Drive, Danvers, MA 01923; include the code 0731-5090/06 $\$ 10.00$ in correspondence with the CCC.

*Staff Engineer, MS 198-326, Guidance and Control Analysis Group, 4800 Oak Grove Drive; paul.b.brugarolas@jpl.nasa.gov. Senior Member AIAA.

'Senior Research Scientist, MS 198-326, Guidance and Control Analysis Group, 4800 Oak Grove Drive; david.s.bayard@jpl.nasa.gov. Senior Member AIAA.

${ }^{\ddagger}$ Senior Engineer, MS 198-326, Guidance and Control Analysis Group, 4800 Oak Grove Drive; john.t.spanos@jpl.nasa.gov.

${ }^{\S}$ Principal Engineer, MS 198-326, Guidance and Control Analysis Group, 4800 Oak Grove Drive; william.g.breckenridge@jpl.nasa.gov. tenna. The laser rangefinder measured the relative distance between the shuttle and a corner cube mounted on the outboard antenna. The mission configuration and geometry are shown in Fig. 1, and the payload configuration is depicted in Fig. 2.

Achieving the objective of creating topographic maps with an absolute height error of $16 \mathrm{~m}$ every $30-\mathrm{m}$ postings required estimating the position of the shuttle with respect to the ground at the $1-\mathrm{m}$ level, the orientation of the antennas to the 1 -arcsecond level, and the relative position/orientation of the antennas to the $1-\mathrm{mm} / 100$ arcsecond levels. A general overview of the calibration and estimation algorithms used to process the AODA measurements to the desired accuracies, is given in Refs. 1 and 2. A description of the SRTM mast is given in Ref. 3, and a study on the damper failure is given in Ref. 4 . Figures 3 and 4 show pictures of the mast taken during the mission.

This paper presents a study to identify a model for the nonlinear roll-bending mode of the $60-\mathrm{m}$ mast. Postmission datareconstruction efforts indicated that the linear model used by the Kalman filter for this particular mode was not sufficient for accurately tracking its transient behavior and that a higher-fidelity nonlinear model was needed. This nonlinear model will be used in a Kalman filter, ${ }^{1,2}$ which estimates the relative position and orientations of the two antennas. A preliminary version of this work appeared in Ref. 5.

\section{Kalman Filter for Relative Position and Attitude}

In this section, we briefly describe the Kalman filter used for estimating the relative position and attitude between the radar antennas. The reader is referred to Ref. 1 for more details. A postflight calibration and tuning of the estimator was presented in Ref. 2. This estimator uses measurements from the ASTROS Target Tracker (ATT), which looks at three LEDs mounted in a triangular configuration at the outboard antenna and an electronic distance meter (rangefinder), and it was implemented as a discrete-time Kalman filter.

The state of the filter was chosen to include six biases, 10 modal states, and one time latency state. The bias states (three for position and three for orientation) estimate misalignments, launch shifts, mast deployment errors, nonlinear sticktion, and thermal distortions. These states do not have dynamics, but we assign them some process noise to track slow changes caused by thermal distortions and sticktion of the roll bending mode. The modal states are the magnitudes and rates of the five lower-frequency flexible modes of the $60-\mathrm{m}$ boom. These are the first and second roll and yaw bending modes and the twist mode. The dynamics for each mode are modeled as second-order systems, where the mode shapes were taken from a NASTRAN model of the shuttle with the SRTM payload (boom deployed, no viscous dampers). The frequency and damping 
of each mode were identified postflight from the flight data. The first roll bending mode showed some nonlinear behaviors, which are the subject of the present paper. Process noise in the rate states were used to account for variations in the frequencies and dampings and inaccuracies on the mode shapes. The latency state estimates an internal time-varying latency in the rangefinder, which is not included in its measurement time tag. This latency state does not have any dynamics, but it has a small process noise to account for variations in the latency. Recorded time histories of the shuttle vernier thrusters were used as the excitation for the Kalman-filter dynamics.

The Kalman filter was formulated in the predictor-corrector as follows:

$$
\begin{aligned}
& \hat{x}(k+1 \mid k+1)=\hat{x}(k+1 \mid k)+K(k+1) r(k+1 \mid k) \\
& \hat{X}(k+1 \mid k+1)=[I-K(k+1) H(k+1)] \hat{X}(k+1 \mid k) \\
& \quad \times[I-K(k+1) H(k+1)]^{T}+K(k+1) V(k+1) K(k+1)^{T}
\end{aligned}
$$

where, the estimator gain $K$ and the innovation process $r$ are defined as

$$
\begin{aligned}
& K(k+1)=\hat{X}(k+1 \mid k) H^{T}(k+1)[H(k+1) \\
& \left.\quad \times \hat{X}(k+1 \mid k) H(k+1)^{T}+V(k+1)\right]^{-1} \\
& r(k+1 \mid k)=z(k+1)-H(k+1) \hat{x}(k+1 \mid k) \text { for } k=0,1,2, \ldots
\end{aligned}
$$
and $H$ and $V$ are the measurement coefficient matrix and measurement noise covariance, respectively.

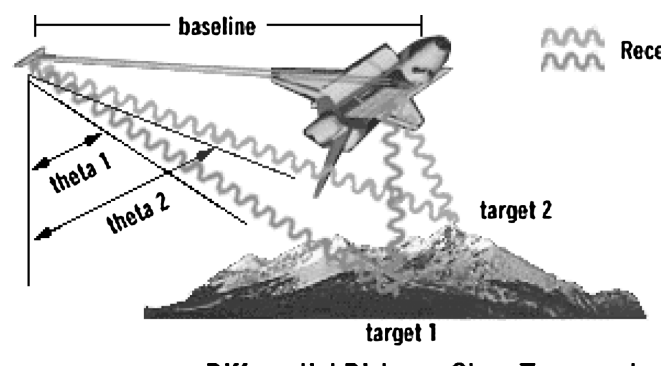

Differential Distance Gives Topography

Fig. 1 SRTM interferometric radar.

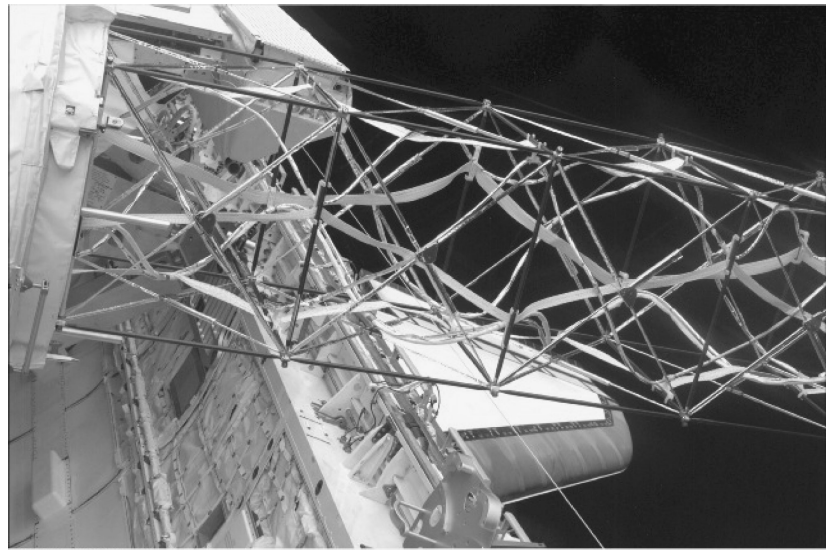

Fig. 3 View of the mast from the shuttle during the mission (courtesy of NASA).

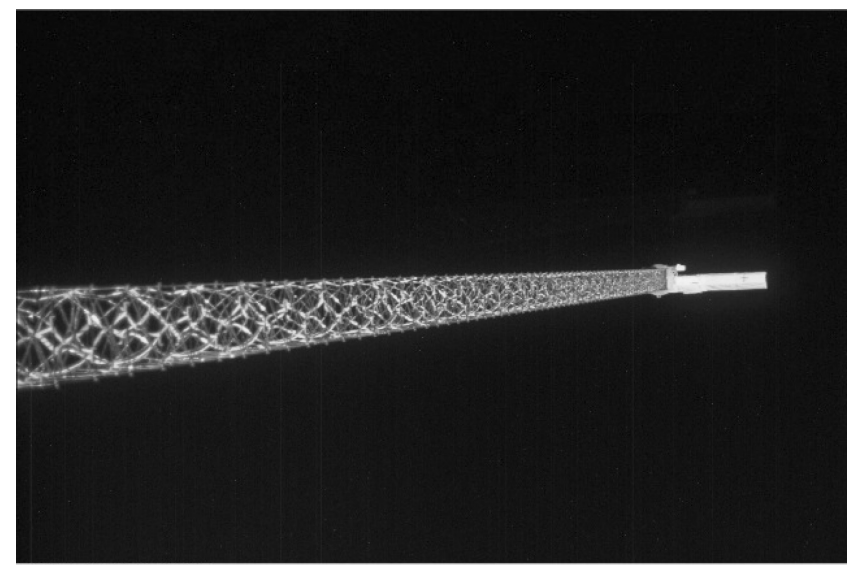

Fig. 4 View of the mast and outboard antenna from the shuttle (courtesy of NASA).

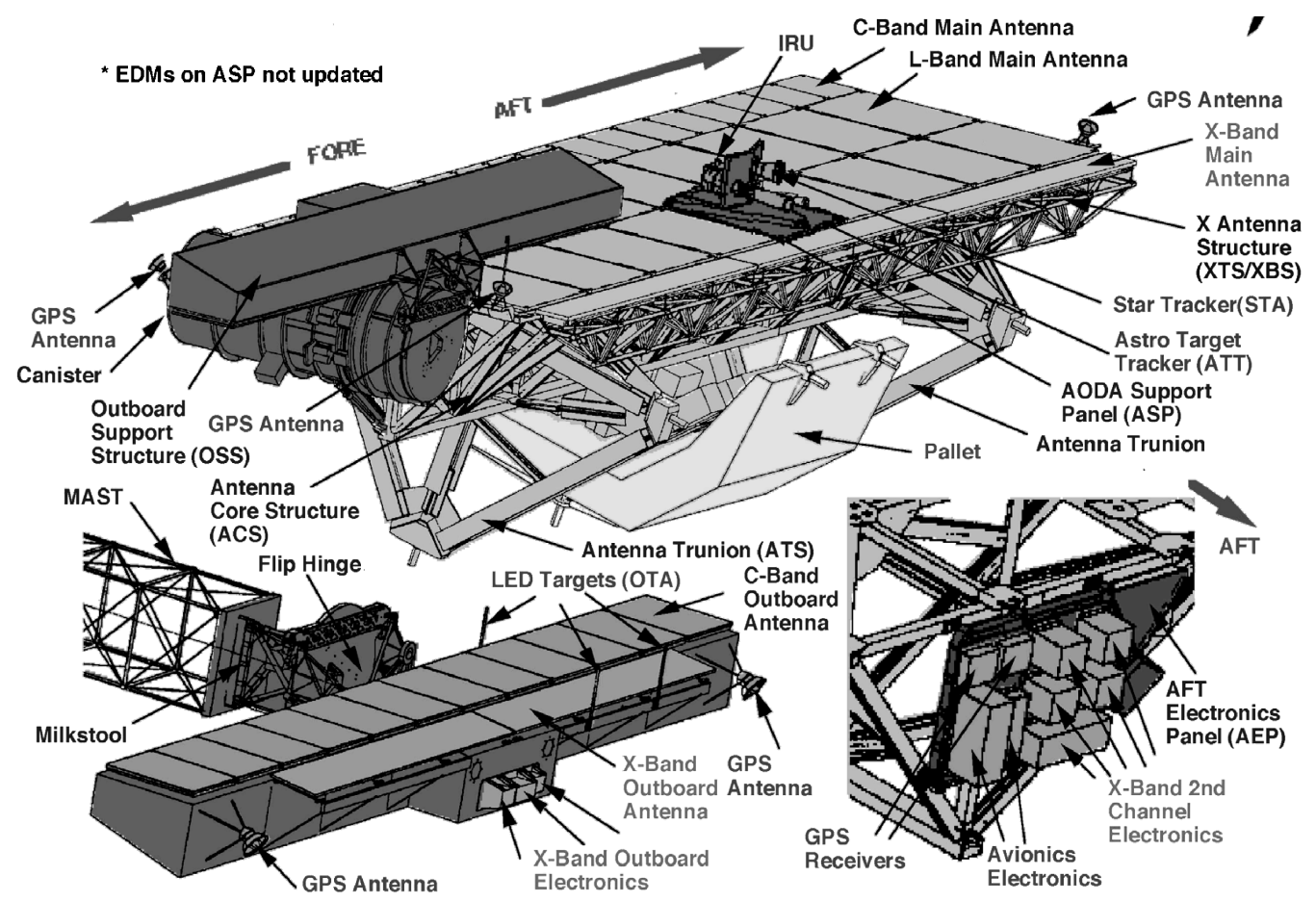

Fig. 2 SRTM attitude and orbit determination avionics. 
The outboard estimator performance was evaluated and manually tuned by studying radar calibration of height errors over ocean passes. (Oceans are relatively flat compared to land, and more readily used for performance evaluation.) As a result of these studies, the estimator was tuned with increased process noise so as to mainly follow the measurements. The state equations were propagated from thruster firings times to measurement update times dictated by the data. Because of inaccuracies in the mode shapes and models, the estimator was set to output estimates at ATT measurements times. Even though thruster firing information was available, it was not sufficiently accurate to improve state propagation errors. However, it was used instead in the covariance propagation equations to indicate the use of increased process noise at times of thruster firing.

\section{Problem Description: Nonlinear Bending Mode}

The first roll bending mode was continually excited by the shuttle attitude control thruster firings, which occurred (very roughly) every $100 \mathrm{~s}$. The induced transient in response to each firing demonstrated a nonlinear behavior.

The nonlinear behavior is apparent from Fig. 5, which shows the ATT measurements of the mast motion. The ATT is a camera mounted on the inboard antenna, which took pictures of three LEDs mounted on the outboard antenna in a triangular configuration. Figure 5 shows the motion in the vertical axis of an LED with respect to time. The vertical axis is more sensitive than the horizontal axis with respect to the roll-bending-mode behavior.

The nonlinear behavior was found to manifest itself predominantly in three ways:

1) The frequency of the decaying sinusoid-like response is amplitude dependent. At high amplitude it tends to oscillate at a higher frequency, with at least a $10 \%$ frequency variation in going from high to low amplitude over a given transient response.

2) The damping is characterized by an envelope that decays with a linear trend (rather than exponential).

3) The mean value over each cycle tends to have two different values: one occurring at medium to high amplitudes and a second when the amplitude dropped below a certain threshold.

The Kalman filter used a linear model of the mast flexible dynamics, extracted from a NASTRAN model of the combined shuttle/ SRTM payload in the radar-mapping configuration (mast deployed, dampers caged). This model included the five lowest frequency modes of the mast, that is, the first and second roll and yaw bending modes, and the twist mode. Each of these modes was modeled as a second-order linear system. This implied that the modal frequency was constant and the damping envelope was exponential. Such linear responses proved to be adequate for four of the five modes in the model. However, the behavior of the first roll bending mode was sufficiently nonlinear to warrant further consideration.

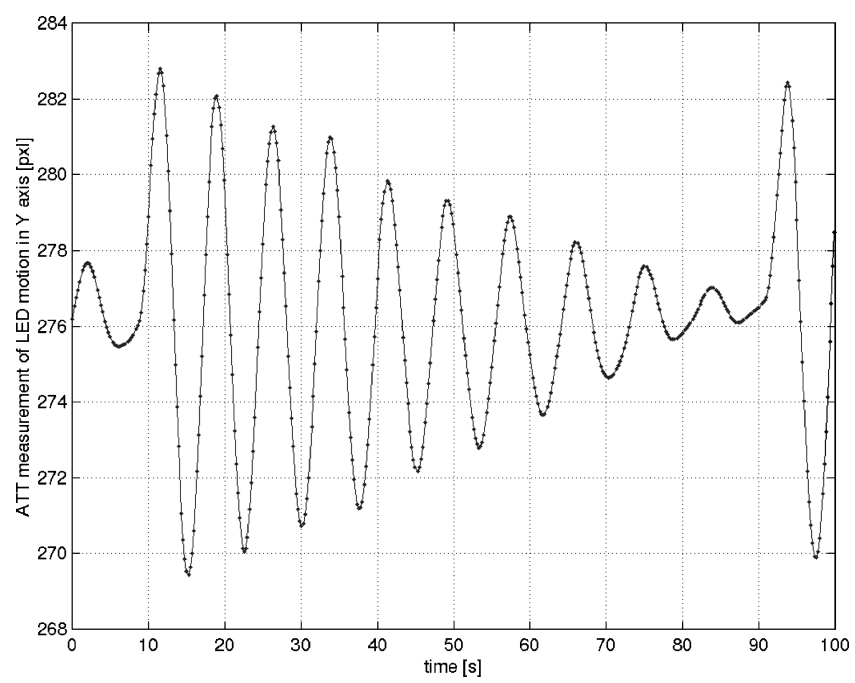

Fig. 5 Nonlinear roll bending mode as seen by the ATT.
Of the nonlinear behaviors just listed, 2 and 3 could be adequately accommodated by moderately increasing the Kalman-filter process noise covariance. However, behavior 1, the frequency-amplitude nonlinearity, was particularly problematic. Therefore, a model for the frequency-amplitude nonlinearity was desired to help achieve the desired performance.

\section{Empirically Determining the Frequency-Amplitude Curve}

An experimental realization of the frequency-amplitude curve was obtained by first running the Kalman filter to reconstruct the modal position and velocity of the roll bending mode (these are specific states estimated by the Kalman filter) and then running a moving windowed least-squares estimator on the position state as shown in Fig. 6.

For the moving windowed estimator, at each time instant, a time window was centered at that point in time and was taken to have a duration of about $90 \%$ of the average period of the roll bending mode. Within this window a best-fit sinusoid (phase, amplitude, and frequency) was determined by numerically minimizing an unweighted least-squares error. Then, this window was moved by about $2 \%$ of the average period, and another best-fit sinusoid was determined, and so forth. An experimentally generated plot of modal amplitude vs squared frequency $\left(\mathrm{rad}^{2} / \mathrm{s}^{2}\right)$ as determined by the moving window technique is shown in Fig. 7. This curve was generated using

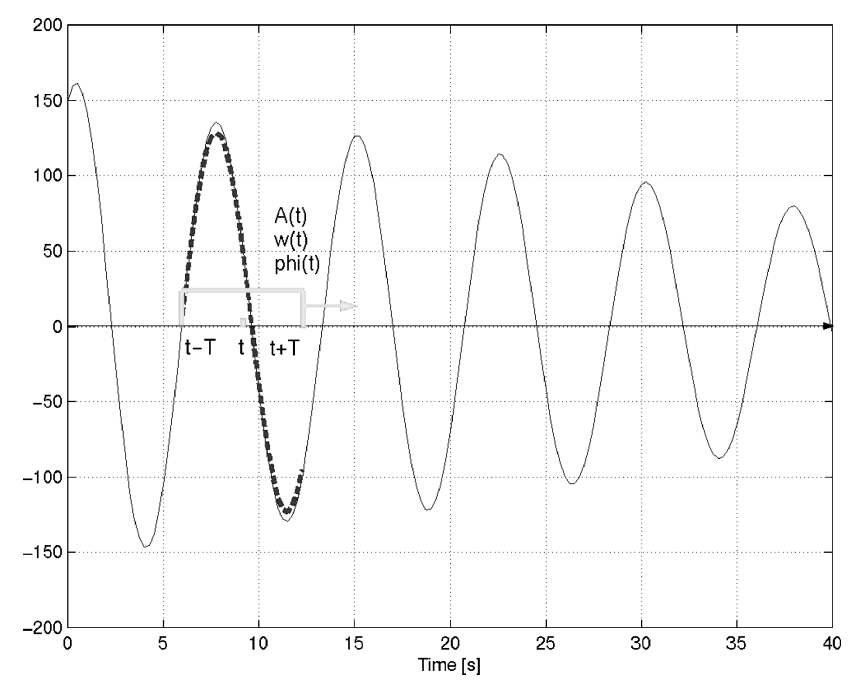

Fig. 6 Moving windowed estimator.

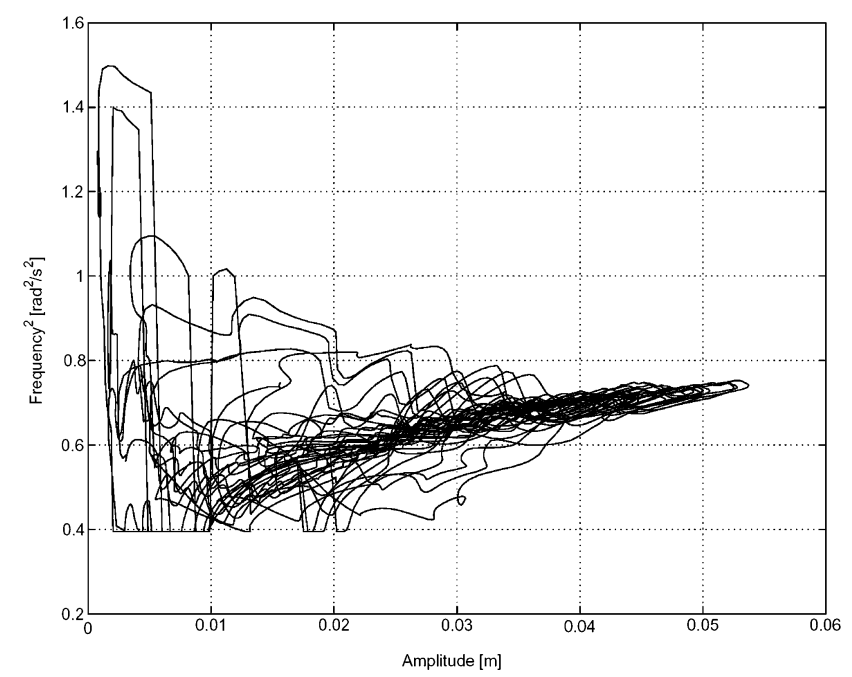

Fig. 7 Empirically determined frequency-amplitude curve of the roll bending mode. 
data that spanned several hours and contained multiple transients (in response to thruster firings).

Figure 7 is very revealing. If the mode were truly linear, all responses would lie on a flat horizontal line at the mode frequency. Clearly this is not the case, and the modal frequency is seen to be an increasing function of the modal amplitude. Specifically, for most traces at low amplitude the frequency starts out in the vicinity of $0.5 \mathrm{rad}^{2} / \mathrm{s}^{2}$ (corresponding to $0.11 \mathrm{~Hz}$ ) and grows to about $0.7 \mathrm{rad}^{2} / \mathrm{s}^{2}$ (corresponding to $0.13 \mathrm{~Hz}$ ). The figure gives the impression that the mode might be suitably modeled with a nonlinear spring, which becomes stiffer as the excursions become greater. Specific parameterizations for identification will be discussed next. The jitter is because the moving window estimator has a transient at the transition between vibration cycles.

\section{System Identification}

Three models were considered for capturing frequency-amplitude nonlinearity. All of the models considered are nonlinear but have linear regression structures. The general usefulness of such an approach to nonlinear identification is discussed in Ljung. ${ }^{6}$

The three models are denoted as the amplitude-dependentstiffness (ADS) model, the Duffing model, and the momentexpansion model. These models are discussed in more detail in the following.

The data used to identify the Duffing and moment expansion models were extracted as follows. The Kalman filter using a linear damping model was run with very large process noise, and the resulting estimates (taken at the ATT measurement times) were used for system identification. With this approach the state estimates closely followed the raw ATT data and are desensitized with respect to the details of the dynamics. The Kalman filter performs the bandpass filtering needed to isolate the modal response and provides smoothed velocity estimates compared to what could be obtained by differencing the position measurements directly.

\section{A. Amplitude-Dependent Stiffness Model}

This model is specified by the nonlinear second-order differential equation

$$
\ddot{x}+\xi \dot{x}+K(x, \dot{x}) x=0
$$

where the stiffness is characterized by the following nonlinear function of the modal amplitude:

$$
K(x, \dot{x})=a+b A(x, \dot{x})+c A(x, \dot{x})^{2}
$$

with parameters $a, b, c$.

The parameters are identified based on fitting empirically generated frequency-amplitude data as determined by the moving window technique discussed earlier. Specifically, let the frequency-squared variable (associated with the vertical axis of Fig. 7) be denoted as $\omega^{2}(t)$ and the amplitude variable (associated with the horizontal axis of Fig. 7) be denoted as $A(t)$.

Then a linear least-squares problem is posed as follows:

$$
\min _{a, b, c}\left[w^{2}(t)-K(t)\right]^{2}=\min _{a, b, c}\left[w^{2}(t)-a-b A(t)-c A^{2}(t)\right]^{2}
$$

The optimal parameters were determined as

$$
a=0.282855, \quad b=1.151121, \quad c=-0.714041
$$

Figure 8 shows the identified ADS model superimposed on one of the experimentally determined frequency-amplitude traces. It is seen to be a reasonable match to the data.

To use the identified model as part of the inboard Kalman filter, an expression for the modal amplitude $A(t)$ is required as an explicit function of the modal position and state. The following expression was developed, motivated by its analogy to the modal amplitude of a linear harmonic oscillator:

$$
A(x, \dot{x})=\sqrt{x^{2}+[\dot{x} / \bar{K}(x, \dot{x})]^{2}}
$$

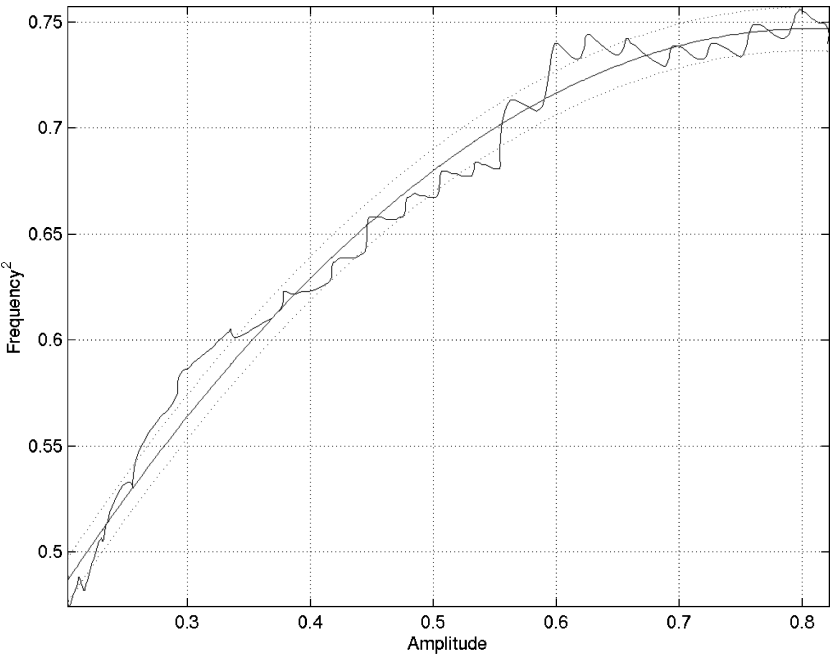

Fig. 8 Frequency-amplitude characteristics of the identified ADS model, superimposed on the average trace from Fig. 6 data.

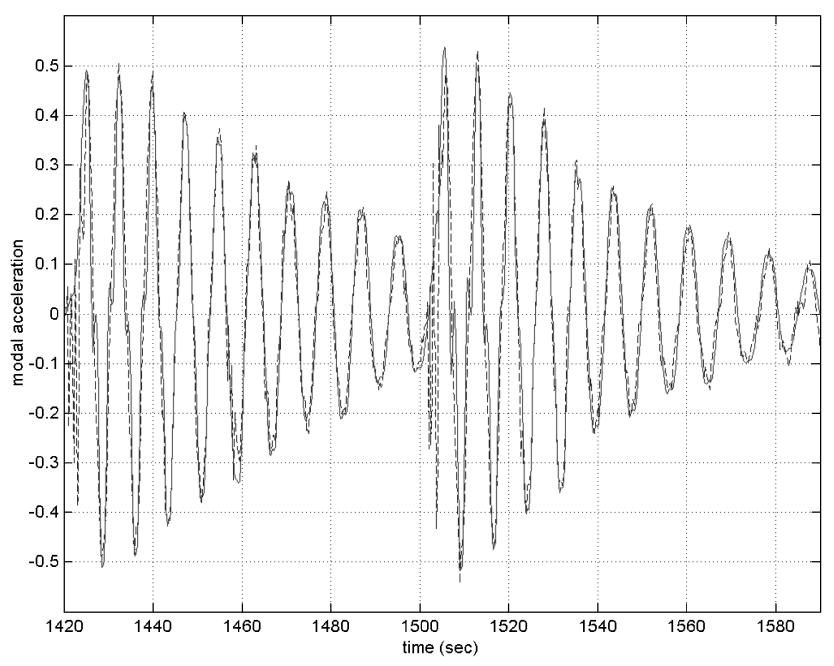

Fig. 9 ADS model response (- $\longrightarrow$ ) superimposed on the Kalman-filterderived modal acceleration (--.--).

Here, the quantity $\bar{K}(x, \dot{x})$ is any reasonable approximation to the stiffness $K(x, \dot{x})$. The approximation finally used was to iterate once, starting with $\bar{K}(x, \dot{x})=a$, and then using the amplitude $A$ thus obtained from Eq. (3) to recalculate a better value for $\bar{K}(x, \dot{x})$ using Eq. (2).

Figure 9 shows the identified ADS model response over a 170-s time period, superimposed on the Kalman-filter-derived modal acceleration. It is seen to be a reasonable match to the data.

\section{B. Duffing Model}

The Duffing model $^{7}$ is specified by the nonlinear second-order differential equation

$$
\ddot{x}+\xi \dot{x}+K(x) x=0
$$

where the stiffness is a nonlinear function of the modal position only, that is,

$$
K(x)=a+b|x|+c x^{2}
$$

with parameters $\xi, a, b, c$.

In contrast to the previous model that was identified based on preprocessed frequency-amplitude data, the parameters of the Duffing model are identified from the modal position and velocity estimates directly obtained from the Kalman-filter states. Specifically, let the Kalman-filter state estimates associated with the roll-bending-mode 


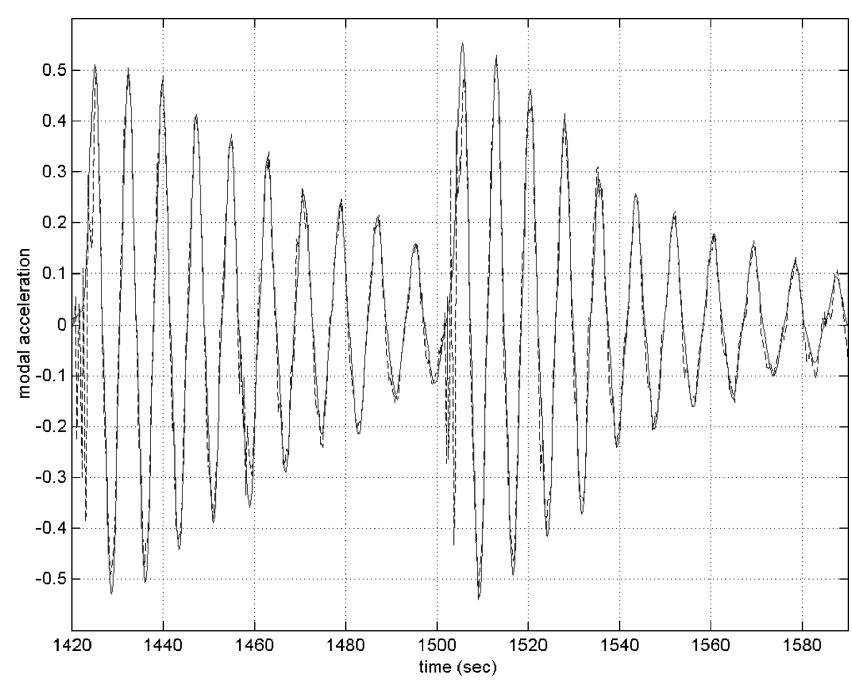

Fig. 10 Duffing model response (-) superimposed on the Kalmanfilter-derived modal acceleration (-- - -).

position and velocity be denoted as $x$ and $\dot{x}$, respectively, and let the acceleration $\ddot{x}$ be derived by differencing the velocity. Then the Duffing model parameters are identified by solving the following unweighted least-squares problem:

$$
\min _{\xi, a, b, c}[\ddot{x}+\xi \dot{x}+K(x) x]^{2}=\min _{\xi, a, b, c}\left[\ddot{x}+\xi \dot{x}+\left(a+b|x|+c x^{2}\right) x\right]^{2}
$$

The optimal parameters were determined as

$$
\xi=0.051, \quad a=0.2731, \quad b=1.407143, \quad c=-1.002551
$$

Figure 10 shows the identified Duffing model response over a 170-s time period, superimposed on the modal acceleration derived from the ATT data. It is seen to be a reasonable match to the data.

\section{Moment-Expansion Model}

This model is specified by the nonlinear second-order differential equation

$$
\ddot{x}+M(x, \dot{x})=0
$$

where a moment expansion is used to capture the damping and stiffness operators as a general nonlinear function of state, that is,

$$
M(x, \dot{x})=\sum_{j=0}^{3} \sum_{\substack{i=0 \\ n o: i=0, j=0}}^{3} p_{i j} x^{i} \dot{x}^{j}
$$

with parameters

$$
p_{i j}\left\{\begin{array}{l}
i=0, \ldots, 3 \\
j=0, \ldots, 3
\end{array} \quad \text { with no } \quad(i, j)=(0,0) \quad\right. \text { term }
$$

As in the Duffing model, the parameters of the moment expansion model are identified from the modal position and velocity estimates directly obtained from the Kalman-filter states and using derived accelerations. Specifically, the moment-expansion (ME) model parameters are identified by solving the following unweighted leastsquares problem over segments of data that do not include thruster firings.

$$
\min _{p_{i j}}[\ddot{x}+M(x, \dot{x})]^{2}=\min _{p_{i j}}\left(\ddot{x}+\sum_{\substack{j=0 \\ n o: i=0, j=0}}^{3} \sum_{\substack{i=0 \\ n o: i}}^{3} p_{i j} x^{i} \dot{x}^{j}\right)^{2}
$$

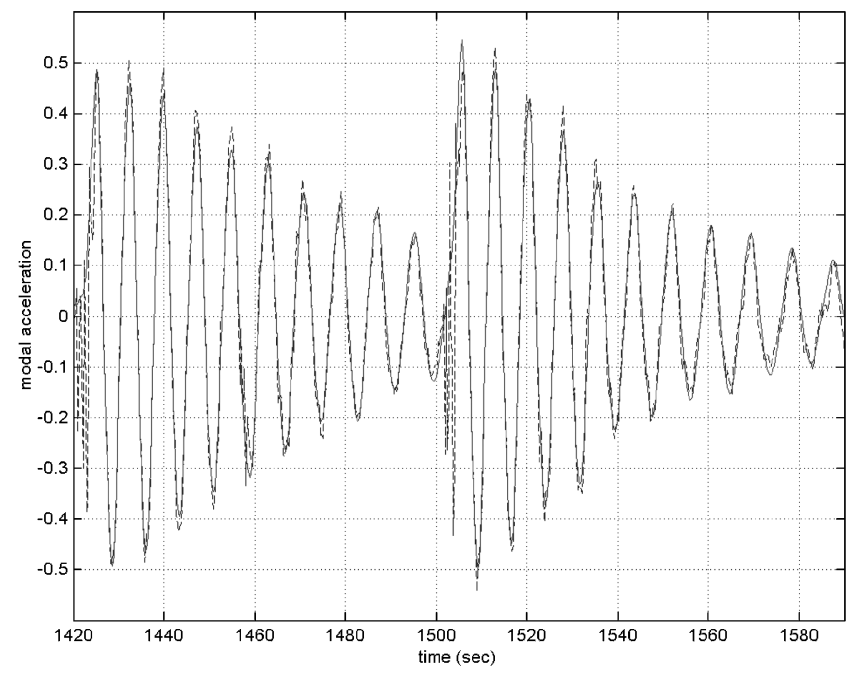

Fig. 11 ME model response (-) superimposed on the Kalmanfilter-derived modal acceleration (--.--).

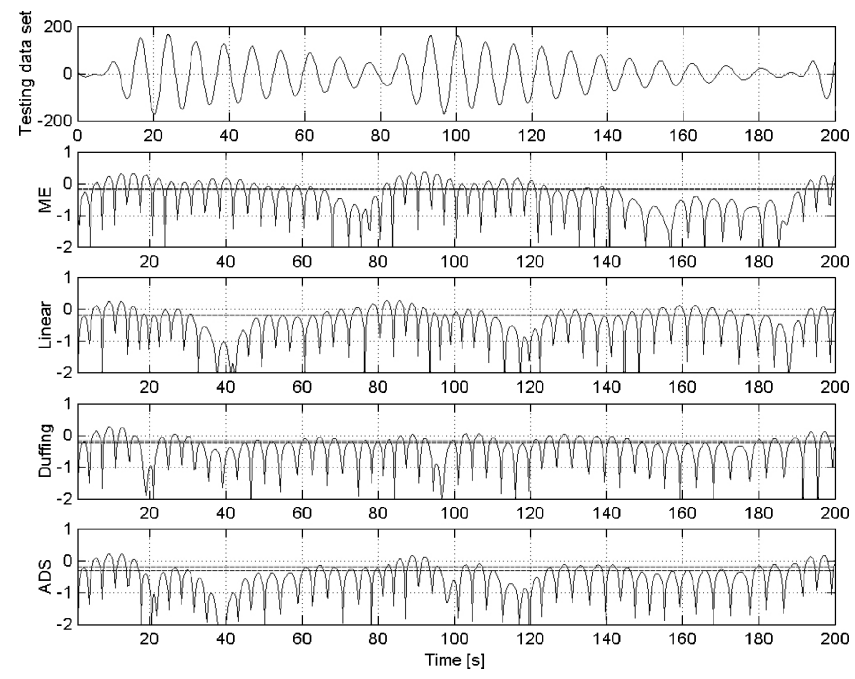

Fig. 12 Testing data set and errors for each of the models.

The optimal parameters were determined as

$$
\begin{array}{ccc}
p_{01}=0.078883 & p_{11}=-0.094683 & p_{21}=-0.182583 \\
p_{10}=0.575785 & p_{20}=0.013407 & p_{12}=0.137373 \\
p_{02}=-0.020779 & p_{30}=0.306406 & p_{03}=0.166841
\end{array}
$$

Figure 11 shows the moment expansion model response superimposed on a 200 -s segment of data measured by the ATT. It is seen to be a reasonable fit to the data.

\section{Validation}

Each of the three models was validated and compared by implementing them in the Kalman filter and studying the rms a priori residuals (in the $0.12-0.14 \mathrm{~Hz}$ frequency band) and the log-likelihood of each alternative model. Although choosing a "best" model based on the smallest rms a priori residuals is closely related to a maximum likelihood criteria, the rms residual criteria have a convenient physical interpretation of being proportional to an rms surface height prediction error, an important figure of merit used by the SRTM mission.

This discussion will focus only on the $z$ component of the mast tip deformation vector, because the SRTM height geometry was most sensitive in this direction. Figure 12 shows plots of a segment of the testing data set and the errors given by each of the models. Each plot also indicates the particular method's rms error using a horizontal 
Table 1 Model performance comparison

\begin{tabular}{lcc}
\hline \hline Method & RMS & Log-likelihood \\
\hline ME model & 0.686 & 3062 \\
Linear model & 0.653 & 2774 \\
Duffing model & 0.561 & 3320 \\
ADS model & 0.471 & 3497 \\
\hline \hline
\end{tabular}

line and includes the rms line associated with the linear model in red for comparison.

The log-likelihood of the $z$ component of the deformation vector of the mast tip is calculated as follows ${ }^{6}$ :

$$
l=c-\frac{1}{2} \sum_{j=1}^{N}\left[\ln \Gamma_{z}(j)+v_{z}(j)^{2}\right]
$$

where $c$ is a constant, $v_{z}(j)=e_{z}(j) / \sqrt{ }\left[\Gamma_{z}(j)\right]$ is the normalized a priori residual of the $z$ component, and $\Gamma_{z}(j)=H_{z} P(j) H_{z}^{T}+R_{z}$ is the a priori residual covariance.

Results for the original linear model, amplitude-dependentstiffness (ADS) model, the Duffing model, and the momentexpansion (ME) model are shown in Table 1. The second column corresponds to the rms value of the a priori residuals for each of the models, as transformed to an equivalent height error over the ground in meters. The third column corresponds to the log-likelihood value of each alternative model, based on using Eq. (8).

It can be seen that all of the nonlinear models performed better than the linear model, in terms of their log-likelihood (the higher, the better). Except for the ME model, the nonlinear models also performed better than the linear model in the rms sense.

The best performance in the sense of both rms and log-likelihood was achieved by the amplitude-dependent-stiffness model. The improvement relative to the linear model was about $20 \mathrm{~cm}$ of rms height error, which was significant and could be visibly discerned from comparing traces. The improvement of the ADS model relative to the Duffing model however was less dramatic, probably because both models were nonlinear and able to capture the amplitudedependent frequency characteristics of the modal response. The moment-expansion model performed worst of all models in an rms residual sense. In retrospect, the problem appeared to be that the dynamic range of the Kalman-filter states extended beyond the range over which the moment expansion model was identified, that is, the identified model did not "generalize" sufficiently well to accommodate this difference. Interestingly, despite a higher rms height error, the ME model was still ranked higher than the linear model in terms of the log-likelihood.

\section{Conclusions}

A study that identified a model for the nonlinear bending mode of a 60-m flexible mast in space was presented, in support of the
Shuttle Radar Topography Mission. The nonlinear model was ultimately used to improve performance of a Kalman filter designed to estimate the relative motion of the two radar antennas, leading directly to improvements in postflight height reconstruction accuracy.

Three nonlinear models, specifically the amplitude-dependentstiffness (ADS), Duffing, and moment-expansion (ME) models, and one linear model were identified and evaluated in this study. The ADS model is a new approach that does not seem to have any counterpart in the literature. It was defined by fitting the empirically determined amplitude-frequency characteristics, rather than the more standard approach of fitting position-velocity data.

The performance of the three models was compared based on rms a priori residual errors and log-likelihood values. It was found that all nonlinear models had a higher log-likelihood than the linear model. The best performance with respect to both rms and log-likelihood criteria was achieved by the ADS model, with a reduction of about $20-\mathrm{cm}$ of rms height error. This improvement was significant, and the ADS model was subsequently used to generate all of the official data products as part of the SRTM postflight reconstruction effort.

\section{Acknowledgments}

This work was carried out at the Jet Propulsion Laboratory, California Institute of Technology, under contract with NASA. The authors thank Ed Wong, Dhemetrios Boussalis, Harry Balian, Gurkirpal Singh, and Jeff Umland of Jet Propulsion Laboratory for many helpful technical discussions.

\section{References}

${ }^{1}$ Wong, E., Breckenridge, W., Boussalis, D., and Brugarolas, P., "Attitude Determination for the Shuttle Radar Topography Mission," AIAA Paper 99-3968, Aug. 1999.

${ }^{2}$ Wong, E., Breckenridge, W., Boussalis, D., Brugarolas, P., Bayard, D. S., Spanos, J., and Singh, G., "Post-Flight Attitude Reconstruction for the Shuttle Radar Topography Mission," Advances in the Astronautical Sciences, Vol. 109, 2001, pp. 213-235.

${ }^{3}$ Gross, D., and Messner, D., "The ABLE Deployable Articulated Mast-Enabling Technology for the Shuttle Radar Topography Mission," Proceedings of the 33rd Aerospace Mechanisms Symposium, May 1999, pp. $15-30$

${ }^{4}$ Umland, J., "SRTM Mast Damping Subsystem Design and Failure Investigation," Proceedings of the 35th Aerospace Mechanisms Symposium, May 2001, pp. 83-94.

${ }^{5}$ Brugarolas, P. B., Bayard, D. S., Spanos, J. T., and Breckenridge, W. G., "System Identification of a Nonlinear Bending Mode for the Shuttle Radar Topography Mission,” AIAA Paper 2002-4937, Aug. 2002.

${ }^{6}$ Ljung, L., System Identification: Theory for the User, Prentice-Hall, Englewood Cliffs, NJ, 1987, Chap. 5 and 7.

${ }^{7}$ Braun, S., and Feldman, M., "Time-Frequency Characteristics of NonLinear Systems," Journal of Mechanical Systems and Signal Processing, Vol. 11, No. 4, 1997, pp. 611-620. 